\title{
ON A QUESTION OF A. E. NUSSBAUM ON MEASURABILITY OF FAMILIES OF CLOSED LINEAR OPERATORS IN A HILBERT SPACE
}

\author{
FRITZ GESZTESY, ALEXANDER GOMILKO, FEDOR SUKOCHEV, AND YURI TOMILOV \\ Dedicated to the memory of A. E. Nussbaum (1925-2009)
}

\begin{abstract}
The purpose of this note is to answer a question A. E. Nussbaum formulated in 1964 about the possible equivalence between weak measurability of a family of densely defined, closed operators $\{T(t)\}_{t \in \mathbb{R}}$ in a separable complex Hilbert space $\mathcal{H}$ on one hand, and the notion of measurability of the $2 \times 2$ operator-valued matrix of projections $\left\{\left(P(\Gamma(T(t)))_{j, k}\right)_{1 \leqslant j, k \leqslant 2}\right\}_{t \in \mathbb{R}}$ onto the graph $\Gamma(T(t))$ of $T(t)$ on the other, in the negative.

We also consider related questions pertaining to the family of adjoint operators $\left\{T(t)^{*}\right\}_{t \in \mathbb{R}}$ and to the issue of whether or not the corresponding maximally defined operator $\mathcal{T}$ in $L^{2}(\mathbb{R} ; d t ; \mathcal{H})$ given by

$$
\begin{aligned}
& (\mathcal{T} f)(t)=T(t) f(t), \quad t \in \mathbb{R}, \\
& f \in \operatorname{dom}(\mathcal{T})=\left\{g \in L^{2}(\mathbb{R} ; d t ; \mathcal{H}) \mid g(t) \in \operatorname{dom}(T(t)) \text { for a.e. } t \in \mathbb{R},\right. \\
& \left.t \mapsto T(t) g(t) \text { is weakly measurable, } \int_{\mathbb{R}}\|T(t) g(t)\|_{\mathcal{H}}^{2} d t<\infty\right\} .
\end{aligned}
$$

is densely defined. Our results demonstrate an interesting distinction between the operator $\mathcal{T}$ and the direct integral $\boldsymbol{T}=\int_{\mathbb{R}}^{\oplus} T(t) d t$ in $L^{2}(\mathbb{R} ; \mathcal{H})$ (the latter requires the additional assumption of measurability of the matrix of projections $\left\{\left(P(\Gamma(T(t)))_{j, k}\right)_{1 \leqslant j, k \leqslant 2}\right\}_{t \in \mathbb{R}}$.

We also provide explicit criteria for the measurability of the matrix of projections $\left\{\left(P(\Gamma(T(t)))_{j, k}\right)_{1 \leqslant j, k \leqslant 2}\right\}_{t \in \mathbb{R}}$.
\end{abstract}

\section{INTRODUCTION}

To briefly set the stage for this note, let $\mathcal{H}$ be a separable complex Hilbert space, and consider the Hilbert space $L^{2}(\mathbb{R} ; d t ; \mathcal{H})$, in short, $L^{2}(\mathbb{R} ; \mathcal{H})$, consisting of equivalence classes $f$ of weakly (and hence strongly) Lebesgue measurable $\mathcal{H}$ valued elements $f(\cdot) \in \mathcal{H}$ (whose elements are equal a.e. on $\mathbb{R}$ ), such that $\|f(\cdot)\|_{\mathcal{H}} \in$ $L^{2}(\mathbb{R} ; d t)$. Of course, $L^{2}(\mathbb{R} ; \mathcal{H})$ can be identified with the constant fiber direct integral $\int_{\mathbb{R}}^{\oplus} \mathcal{H} d t$, that is,

$$
L^{2}(\mathbb{R} ; \mathcal{H})=\int_{\mathbb{R}}^{\oplus} \mathcal{H} d t
$$

Date: August 23, 2018.

2000 Mathematics Subject Classification. Primary 46E40, 47A05, 47B40; Secondary 47B80, $46 \mathrm{C} 05$.

Key words and phrases. Closed linear operators, spectral operators, direct integrals.

A.G. and Y.T. were partially supported by the Marie Curie "Transfer of Knowledge" programme, project "TODEQ". Y.T. was also partially supported by a MNiSzW grant Nr. N201384834. The work of F.S. was partially supported by the ARC.. 
Let $\mathbb{R} \ni t \mapsto g(t) \in \mathcal{H}$, then a family $\{g(t)\}_{t \in \mathbb{R}}$ is called weakly measurable in $\mathcal{H}$ if $\mathbb{R} \ni t \mapsto(h, g(t))_{\mathcal{H}}$ is (Lebesgue) measurable for each $h \in \mathcal{H}$.

Throughout this manuscript, boldface calligraphic symbols, such as $\mathcal{S}$, denote operators in the Hilbert space $L^{2}(\mathbb{R} ; \mathcal{H})$ associated with a family of linear operators $\{S(t)\}_{t \in \mathbb{R}}$ in $\mathcal{H}$, maximally defined by

$$
\begin{aligned}
& (\mathcal{S} f)(t)=S(t) f(t) \text { for a.e. } t \in \mathbb{R} \\
& f \in \operatorname{dom}(\mathcal{S})=\left\{g \in L^{2}(\mathbb{R} ; \mathcal{H}) \mid g(t) \in \operatorname{dom}(S(t)) \text { for a.e. } t \in \mathbb{R},\right. \\
& \left.t \mapsto S(t) g(t) \text { is (weakly) measurable, } \int_{\mathbb{R}}\|S(t) g(t)\|_{\mathcal{H}}^{2} d t<\infty\right\} .
\end{aligned}
$$

One readily verifies that if $S(t)$ are closed in $\mathcal{H}$ for a.e. $t \in \mathbb{R}$, then $\mathcal{S}$ is closed in $L^{2}(\mathbb{R} ; \mathcal{H})$.

Next, let $\{T(t)\}_{t \in \mathbb{R}}$ be a family of densely defined, closed, linear operators in $\mathcal{H}$. Then the family $\{T(t)\}_{t \in \mathbb{R}}$ is called weakly measurable if for any weakly measurable family of elements $\{f(t)\}_{t \in \mathbb{R}}$ in $\mathcal{H}$ such that $f(t) \in \operatorname{dom}(T(t))$ for a.e. $t \in \mathbb{R}$, the family of elements $\{T(t) f(t)\}_{t \in \mathbb{R}}$ is weakly measurable in $\mathcal{H}$.

Given the family $\{T(t)\}_{t \in \mathbb{R}}$, one defines the operator $\mathcal{T}$ in $L^{2}(\mathbb{R} ; \mathcal{H})$ as in (1.2). Then $\mathcal{T}$ is closed but not necessarily densely defined. In order to relate $\mathcal{T}$ with the direct integral over the operators $T(t), t \in \mathbb{R}$, with respect to Lebesgue measure, Nussbaum [17] introduces the following fundamental notion of measurability of the

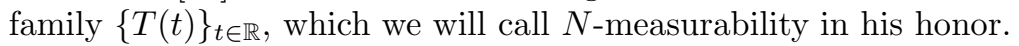

Denote by $\left\{(P(\Gamma(T(t))))_{1 \leqslant j, k \leqslant 2}\right\}_{t \in \mathbb{R}}$ the $2 \times 2$ operator-valued matrix of projections onto the graph $\Gamma(T)$ of $T(t)$. Then the family $\{T(t)\}_{t \in \mathbb{R}}$ is called $N$-measurable if $\left\{\left(P(\Gamma(T(t)))_{j, k}\right\}_{t \in \mathbb{R}}, j, k \in\{1,2\}\right.$, are weakly measurable.

Assuming the family $\{T(t)\}_{t \in \mathbb{R}}$ to be $N$-measurable, the operator $\mathcal{T}$ is called decomposable in $L^{2}(\mathbb{R} ; \mathcal{H})=\int_{\mathbb{R}}^{\oplus} \mathcal{H} d t$ and also denoted by the direct integral of the family $\{T(t)\}_{t \in \mathbb{R}}$ over $\mathbb{R}$ with respect to Lebesgue measure,

$$
\boldsymbol{T}=\int_{\mathbb{R}}^{\oplus} T(t) d t .
$$

Throughout this manuscript, boldface symbols, such as $\boldsymbol{T}$, denote operators in the Hilbert space $L^{2}(\mathbb{R} ; \mathcal{H})$ associated with the direct integral over the family $\{T(t)\}_{t \in \mathbb{R}}$ as depicted in (1.3) (in contrast to our choice of notation $\mathcal{T}$ in the context of $(1.2))$.

Given these preparations, we can now attempt to formulate the question posed by Nussbaum [17, p. 36].

In the special case of bounded operators $T(t) \in \mathcal{B}(\mathcal{H}), t \in \mathbb{R}$, Nussbaum [17] proved the equivalence of $N$-measurability and weak measurability of the family $\{T(t)\}_{t \in \mathbb{R}}$. Moreover, he also proved that in general, $N$-measurability of $\{T(t)\}_{t \in \mathbb{R}}$ implies weak measurability of $\{T(t)\}_{t \in \mathbb{R}}$. However, in the case of unbounded operators $T(t)$, the converse of this fact, and hence the following version of Nussbaum's question:

- Is $N$-measurability of $\{T(t)\}_{t \in \mathbb{R}}$ equivalent to weak measurability of $\{T(t)\}_{t \in \mathbb{R}}$ ?

appears to have been open since 1964 .

The principal purpose of this note is to answer Nussbaum's question in the negative and hence demonstrate an interesting distinction between operators $\mathcal{T}$ in 
$L^{2}(\mathbb{R} ; \mathcal{H})$ defined according to (1.2) on one hand, and direct integrals $\boldsymbol{T}=\int_{\mathbb{R}}^{\oplus} T(t) d t$ as in (1.3), on the other hand, in the sense that it may happen that $\mathcal{T}$ exists but that $\mathcal{T}$ cannot be identified with $\int_{\mathbb{R}}^{\oplus} T(t) d t$.

In addition, we also answer the following natural question:

- Is weak measurability of $\{T(t)\}_{t \in \mathbb{R}}$ equivalent to weak measurability

$$
\text { of }\left\{T(t)^{*}\right\}_{t \in \mathbb{R}} \text { ? }
$$

in the negative (thereby independently answering Nussbaum's question (1.4) in the negative once again). Finally, we also address the question of whether operators of the type $\mathcal{T}$ are densely defined in $L^{2}(\mathbb{R} ; \mathcal{H})$.

In Section 2 we very briefly recall basic facts on closed operators and their graphs following Stone's fundamental paper [23]. Fundamental facts for direct integrals of (unbounded) closed operators as developed in Nussbaum [17] (see also Lennon [13] and Pallu de la Barrière [18]) are summarized in Section 3. In our final Section 4 we present counterexamples to questions (1.3) and (1.4), investigate when $\mathcal{T}$ is densely defined or not, and conclude with a sufficient criterion for a weakly measurable family $\{T(t)\}_{t \in \mathbb{R}}$ to be $N$-measurable in terms of the resolvents of $T(t)$, $t \in \mathbb{R}$.

Finally, we briefly summarize some of the notation used in this paper: Let $\mathcal{H}$ be a separable complex Hilbert space, $(\cdot, \cdot)_{\mathcal{H}}$ the scalar product in $\mathcal{H}$ (linear in the second argument), and $I_{\mathcal{H}}$ the identity operator in $\mathcal{H}$. Next, let $T$ be a linear operator mapping (a subspace of) a Hilbert space into another, with $\operatorname{dom}(T)$ and $\operatorname{ker}(T)$ denoting the domain and kernel (i.e., null space) of $T$. The resolvent set of a closed linear operator in $\mathcal{H}$ will be denoted by $\rho(\cdot)$. The Banach space of bounded linear operators on $\mathcal{H}$ is denoted by $\mathcal{B}(\mathcal{H})$.

\section{Some Facts on Closed linear Operators}

The principal purpose of this short section is to briefly recall some basic facts on closed operators and their graphs discussed in great detail in Stone's fundamental paper [23] (see also von Neumann [26, Ch. XIII, App. II]).

For simplicity, we make the following assumption:

Hypothesis 2.1. Let $\mathcal{H}$ be a complex separable Hilbert space and $T$ a densely defined, closed, linear operator in $\mathcal{H}$.

We note that Stone [23] considers a more general situation, but Hypothesis 2.1 fits the purpose of our paper.

By $\Gamma(T)$ we denote the graph of $T$, that is, the following subspace of the direct $\operatorname{sum} \mathcal{H} \oplus \mathcal{H}$,

$$
\Gamma(T)=\{\langle f, T f\rangle \mid f \in \operatorname{dom}(T)\} \subseteq \mathcal{H} \oplus \mathcal{H} .
$$

Since $T$ is assumed to be closed, $\Gamma(T)$ is a closed subspace of $\mathcal{H} \oplus \mathcal{H}$. Here $\langle f, g\rangle$ denotes the ordered pair of $f, g \in \mathcal{H}$, and we use the standard norm

$$
\|\langle f, g\rangle\|_{\mathcal{H} \oplus \mathcal{H}}=\left[\|f\|_{\mathcal{H}}^{2}+\|g\|_{\mathcal{H}}^{2}\right]^{1 / 2}, \quad f, g \in \mathcal{H},
$$

and scalar product

$$
\left(\left\langle f_{1}, g_{1}\right\rangle,\left\langle f_{2}, g_{2}\right\rangle\right)_{\mathcal{H} \oplus \mathcal{H}}=\left(f_{1}, f_{2}\right)_{\mathcal{H}}+\left(g_{1}, g_{2}\right)_{\mathcal{H}}, \quad f_{j}, g_{j} \in \mathcal{H}, j=1,2,
$$

in $\mathcal{H} \oplus \mathcal{H}$. 
If $B \in \mathcal{B}(\mathcal{H} \oplus \mathcal{H})$, one can uniquely represent $B$ as the $2 \times 2$ block operator matrix

$$
B=\left(\begin{array}{ll}
B_{1,1} & B_{1,2} \\
B_{2,1} & B_{2,2}
\end{array}\right)
$$

where $B_{j, k} \in \mathcal{B}(\mathcal{H}), j, k \in\{1,2\}$.

Denoting by

$$
P(\Gamma(T))=\left(\begin{array}{ll}
P(\Gamma(T))_{1,1} & P(\Gamma(T))_{1,2} \\
P(\Gamma(T))_{2,1} & P(\Gamma(T))_{2,2}
\end{array}\right)
$$

the orthogonal projection onto $\Gamma(T)$, the corresponding matrix $\left(P(\Gamma(T))_{j, k}\right)_{1 \leqslant j, k \leqslant 2}$ will be called the characteristic matrix of $T$. Since by hypothesis $T$ is closed and densely defined, one obtains (cf. [23])

$$
\begin{aligned}
& \left(P(\Gamma(T))_{j, k}\right)^{*}=P(\Gamma(T))_{k, j}, \quad j, k \in\{1,2\}, \\
& \sum_{k=1}^{2} P(\Gamma(T))_{j, k} P(\Gamma(T))_{k, \ell}=P(\Gamma(T))_{j, \ell}, \quad j, \ell \in\{1,2\}, \\
& \operatorname{ker}\left(P(\Gamma(T))_{1,1}\right)=\operatorname{ker}\left(I_{\mathcal{H}}-P(\Gamma(T))_{2,2}\right)=\{0\}, \\
& P\left(\Gamma\left(T^{*}\right)\right)=\left(\begin{array}{cc}
I_{\mathcal{H}}-P(\Gamma(T))_{2,2} & P(\Gamma(T))_{2,1} \\
P(\Gamma(T))_{1,2} & I_{\mathcal{H}}-P(\Gamma(T))_{1,1}
\end{array}\right), \\
& \operatorname{ker}(T)=\{0\} \text { if and only if } \operatorname{ker}\left(I_{\mathcal{H}}-P(\Gamma(T))_{1,1}\right)=\{0\}, \\
& P\left(\Gamma\left(T^{-1}\right)\right)=\left(\begin{array}{cc}
P(\Gamma(T))_{2,2} & P(\Gamma(T))_{2,1} \\
P(\Gamma(T))_{1,2} & P(\Gamma(T))_{1,1}
\end{array}\right) \text { if } \operatorname{ker}(T)=\{0\}, \\
& P(\Gamma(T))_{2,1}=T P(\Gamma(T))_{1,1}, \quad P(\Gamma(T))_{2,2}=T P(\Gamma(T))_{1,2}, \\
& I_{\mathcal{H}}-P(\Gamma(T))_{1,1}=T^{*} P(\Gamma(T))_{2,1}, \quad P(\Gamma(T))_{1,2}=T^{*}\left(I_{\mathcal{H}}-P(\Gamma(T))_{2,2}\right) .
\end{aligned}
$$

In particular, one has the following explicit expressions for $P(\Gamma(T))_{j, k}, j, k \in\{1,2\}$ :

$$
\begin{aligned}
& P(\Gamma(T))_{1,1}=\left(T^{*} T+I_{\mathcal{H}}\right)^{-1}, \\
& P(\Gamma(T))_{1,2}=T^{*}\left(T T^{*}+I_{\mathcal{H}}\right)^{-1}, \\
& P(\Gamma(T))_{2,1}=T\left(T^{*} T+I_{\mathcal{H}}\right)^{-1}, \\
& P(\Gamma(T))_{2,2}=T T^{*}\left(T T^{*}+I_{\mathcal{H}}\right)^{-1}=I_{\mathcal{H}}-\left(T T^{*}+I_{\mathcal{H}}\right)^{-1}
\end{aligned}
$$

(see also [2], [10], and [16] for generalizations to closed linear relations).

\section{Basic Facts on Direct Integrals of Closed Operators}

We briefly recall some facts for direct integrals of unbounded closed operators as developed in Nussbaum [17] (see also Dixon [8], Lennon [13], Pallu de la Barrière [18], and Takemoto [24]).

We study families of densely defined, closed operators $\{T(t)\}_{t \in \mathbb{R}}$ in $\mathcal{H}$ and use the following assumption for the remainder of this section:

Hypothesis 3.1. Let $T(t), t \in \mathbb{R}$, be densely defined, closed, linear operators in $\mathcal{H}$.

We need the following notions of measurable vector and operator families:

Definition 3.2. (i) Let $\mathbb{R} \ni t \mapsto g(t) \in \mathcal{H}$. Then the family $\{g(t)\}_{t \in \mathbb{R}}$ is called weakly measurable in $\mathcal{H}$ if $\mathbb{R} \ni t \mapsto(h, g(t))_{\mathcal{H}}$ is (Lebesgue) measurable for each $h \in \mathcal{H}$. 
Next, assume Hypothesis 3.1:

(ii) The family $\{T(t)\}_{t \in \mathbb{R}}$ is called weakly measurable if for any weakly measurable family of elements $\{f(t)\}_{t \in \mathbb{R}}$ in $\mathcal{H}$ such that $f(t) \in \operatorname{dom}(T(t))$ for all $t \in \mathbb{R}$, the family of elements $\{T(t) f(t)\}_{t \in \mathbb{R}}$ is weakly measurable in $\mathcal{H}$.

(iii) The family $\{T(t)\}_{t \in \mathbb{R}}$ is called $N$-measurable if the entries of the characteristic matrix of $T(t)$ are weakly measurable, that is, if $\left\{P(\Gamma(T(t)))_{j, k}\right\}_{t \in \mathbb{R}}, j, k \in\{1,2\}$, are weakly measurable.

For notational simplicity the vector and operator families in this note are defined for all $t \in \mathbb{R}$ rather than the customary a.e. $t \in \mathbb{R}$ with respect to Lebesgue measure.

We note that measurability of the characteristic matrix $\left(P(\Gamma(T(\cdot)))_{j, k}\right)_{1 \leqslant j, k \leqslant 2}$ of $T(\cdot)$ was introduced by Nussbaum [17]. In fact, he considered the more general situation of a general measure $d \mu$ and a $\mu$-measurable family of Hilbert spaces $\{\mathcal{H}(t)\}_{t \in \mathbb{R}}$.

We summarize a few consequences of Definition 3.2 in Remark 3.3 below:

Remark 3.3. (i) Since $\mathcal{H}$ is assumed to be separable, weak measurability of the family $\{g(t)\}_{t \in \mathbb{R}}$ in $\mathcal{H}$ is equivalent to (strong) measurability, that is, there exists a sequence of countably-valued elements $\left\{g_{n}(t)\right\}_{t \in \mathbb{R}} \subset \mathcal{H}, n \in \mathbb{N}$, and a set $\mathcal{E} \subset \mathbb{R}$ of Lebesgue measure zero such that $\lim _{n \rightarrow \infty}\left\|g_{n}(t)-g(t)\right\|_{\mathcal{H}}=0$ for each $t \in \mathbb{R} \backslash \mathcal{E}$. Thus, the family $\{g(t)\}_{t \in \mathbb{R}}$ is (weakly) measurable in $\mathcal{H}$ if there exists a dense set $\mathcal{Y} \subset \mathcal{H}$ such that the function $(y, g(\cdot))_{\mathcal{H}}$ is measurable for every $y \in \mathcal{Y}$, see, for instance, [1, Corollary 1.1.3], [6, p. 42-43]. Moreover,

$$
f, g: \mathbb{R} \mapsto \mathcal{H} \text { measurable } \Longrightarrow(f(\cdot), g(\cdot))_{\mathcal{H}} \text { is measurable. }
$$

(ii) One can show (cf. [17, Corollary 2]) that

$N$-measurability of $\{T(t)\}_{t \in \mathbb{R}} \Longrightarrow$ weak measurability of $\{T(t)\}_{t \in \mathbb{R}}$.

(The converse, however, is false as we will show in Section 4.)

(iii) Since by $(2.6), P(\Gamma(T(t)))_{2,1}=\left(P(\Gamma(T(t)))_{1,2}\right)^{*}$, or equivalently, since

$$
\begin{aligned}
{\left[T(t)\left(T(t)^{*} T(t)+I_{\mathcal{H}}\right)^{-1}\right]^{*} } & =T(t)^{*}\left(T(t) T(t)^{*}+I_{\mathcal{H}}\right)^{-1} \\
& \supseteq\left(T(t)^{*} T(t)+I_{\mathcal{H}}\right)^{-1} T(t)^{*},
\end{aligned}
$$

as $T(t)$ is closed in $\mathcal{H}$ (this follows from standard properties of adjoints of products of linear operators and from (2.6), (2.9), and (2.14); see also [5, Theorem 2 (ii)]), weak measurability of the operator $\left\{P(\Gamma(T(t)))_{1,2}\right\}_{t \in \mathbb{R}}$ is equivalent to that of $\left\{P(\Gamma(T(t)))_{2,1}\right\}_{t \in \mathbb{R}}$. Thus, by $(2.14)$,

$N$-measurability of $\{T(t)\}_{t \in \mathbb{R}}$ is equivalent to weak measurability of

$$
\begin{aligned}
& \left\{\left(|T(t)|^{2}+I_{\mathcal{H}}\right)^{-1}\right\}_{t \in \mathbb{R}}, \quad\left\{T(t)\left(|T(t)|^{2}+I_{\mathcal{H}}\right)^{-1}\right\}_{t \in \mathbb{R}}, \\
& \text { and }\left\{\left(\left|T(t)^{*}\right|^{2}+I_{\mathcal{H}}\right)^{-1}\right\}_{t \in \mathbb{R}} .
\end{aligned}
$$

(iv) Items (ii) and (iii) show that

$$
N \text {-measurability of }\{T(t)\}_{t \in \mathbb{R}} \Longleftrightarrow N \text {-measurability of }\left\{T(t)^{*}\right\}_{t \in \mathbb{R}} \text {. }
$$

$(v)$ If $T(t) \in \mathcal{B}(\mathcal{H}), t \in \mathbb{R}$, then weak measurability of $\{T(t)\}_{t \in \mathbb{R}}$ is equivalent to

$$
(g, T(t) h)_{\mathcal{H}} \text { is (Lebesgue) measurable for each } g, h \in \mathcal{H} .
$$


Moreover (cf. [17, Proposition 6]),

if $T(t) \in \mathcal{B}(\mathcal{H})$ for a.e. $t \in \mathbb{R}$, then

weak measurability of $\{T(t)\}_{t \in \mathbb{R}} \Longleftrightarrow N$-measurability of $\{T(t)\}_{t \in \mathbb{R}}$.

The Hilbert space $L^{2}(\mathbb{R} ; d t ; \mathcal{H})$, in short, $L^{2}(\mathbb{R} ; \mathcal{H})$, consists of equivalence classes $f$ of weakly (and hence strongly) Lebesgue measurable $\mathcal{H}$-valued elements $f(\cdot) \in \mathcal{H}$ (whose elements are equal a.e. on $\mathbb{R}$ ), such that $\|f(\cdot)\|_{\mathcal{H}} \in L^{2}(\mathbb{R} ; d t)$. The norm and scalar product on $L^{2}(\mathbb{R} ; \mathcal{H})$ are then given by

$$
\|f\|_{L^{2}(\mathbb{R} ; \mathcal{H})}^{2}=\int_{\mathbb{R}}\|f(t)\|_{\mathcal{H}}^{2} d t, \quad(f, g)_{L^{2}(\mathbb{R} ; \mathcal{H})}=\int_{\mathbb{R}}(f(t), g(t))_{\mathcal{H}} d t, \quad f, g \in L^{2}(\mathbb{R} ; \mathcal{H}) .
$$

Of course, $L^{2}(\mathbb{R} ; \mathcal{H})$ can be identified with the constant fiber direct integral $\int_{\mathbb{R}}^{\oplus} \mathcal{H} d t$ (cf., e.g., [7, Sect. II.1], [26, Ch. XII]), that is,

$$
L^{2}(\mathbb{R} ; \mathcal{H})=\int_{\mathbb{R}}^{\oplus} \mathcal{H} d t
$$

We recall our convention that throughout this manuscript, boldface calligraphic symbols, such as $\mathcal{S}$, denote operators in the Hilbert space $L^{2}(\mathbb{R} ; \mathcal{H})$ associated with a family of linear operators $\{S(t)\}_{t \in \mathbb{R}}$ in $\mathcal{H}$, maximally defined by

$$
\begin{aligned}
& (\mathcal{S} f)(t)=S(t) f(t) \text { for a.e. } t \in \mathbb{R}, \\
& f \in \operatorname{dom}(\mathcal{S})=\left\{g \in L^{2}(\mathbb{R} ; \mathcal{H}) \mid g(t) \in \operatorname{dom}(S(t)) \text { for a.e. } t \in \mathbb{R},\right. \\
& \left.t \mapsto S(t) g(t) \text { is (weakly) measurable, } \int_{\mathbb{R}}\|S(t) g(t)\|_{\mathcal{H}}^{2} d t<\infty\right\} .
\end{aligned}
$$

An elementary argument shows that if $S(t)$ are closed in $\mathcal{H}$ for all $t \in \mathbb{R}$, then $\mathcal{S}$ is closed in $L^{2}(\mathbb{R} ; \mathcal{H})$. Indeed, suppose that $\left\{f_{n}\right\}_{n \in \mathbb{N}} \subset \operatorname{dom}(\mathbf{S})$ such that for some $f, g \in L^{2}(\mathbb{R} ; \mathcal{H})$,

$$
\begin{aligned}
\lim _{n \rightarrow \infty} & {\left[\left\|\mathcal{S} f_{n}-g\right\|_{L^{2}(\mathbb{R} ; \mathcal{H})}^{2}+\left\|f_{n}-f\right\|_{L^{2}(\mathbb{R} ; \mathcal{H})}^{2}\right] } \\
\quad & =\lim _{n \rightarrow \infty} \int_{\mathbb{R}} d t\left[\left\|S(t) f_{n}(t)-g(t)\right\|_{\mathcal{H}}^{2}+\left\|f_{n}(t)-f(t)\right\|_{\mathcal{H}}^{2}\right]=0 .
\end{aligned}
$$

Then there exists a subsequence $\left\{f_{n_{m}}\right\}_{m \in \mathbb{N}}$ of $\left\{f_{n}\right\}_{n \in \mathbb{N}}$ such that

$$
\lim _{m \rightarrow \infty}\left[\left\|S(t) f_{n_{m}}(t)-g(t)\right\|_{\mathcal{H}}^{2}+\left\|f_{n_{m}}(t)-f(t)\right\|_{\mathcal{H}}^{2}\right]=0 \text { for a.e. } t \in \mathbb{R} .
$$

Since by hypothesis $S(t)$ is closed in $\mathcal{H}$ for all $t \in \mathbb{R}$, (3.13) implies that for a.e. $t \in \mathbb{R}, f(t) \in \operatorname{dom}(S(t)),\{f(t)\}_{t \in \mathbb{R}}$ is (weakly) measurable in $\mathcal{H}$ (cf. Remark 3.3 $(i)$ ), and $S(t) f(t)=g(t)$, that is, $f \in \operatorname{dom}(\mathcal{S})$ and $\mathcal{S} f=g$, proving that $\mathcal{S}$ is closed.

Thus, assuming Hypothesis 3.1, one infers that $\mathcal{T}$, defined according to (3.10) in terms of the family $\{T(t)\}_{t \in \mathbb{R}}$, is closed in $L^{2}(\mathbb{R} ; \mathcal{H}$ ) (but $\mathcal{T}$ might not be densely defined, cf. Example 4.3 and Remark 4.7). If in addition, the family $\{T(t)\}_{t \in \mathbb{R}}$ is $N$ measurable, then $\mathcal{T}$ is called decomposable in $L^{2}(\mathbb{R} ; \mathcal{H})=\int_{\mathbb{R}}^{\oplus} \mathcal{H} d t$ and also denoted by the direct integral of the family $\{T(t)\}_{t \in \mathbb{R}}$ over $\mathbb{R}$ with respect to Lebesgue measure,

$$
\boldsymbol{T}=\int_{\mathbb{R}}^{\oplus} T(t) d t
$$


(cf. also [7, Ch. II], [15], [22, Ch. I], [25], [26, Ch. XIV] in the context of bounded operators; [4], [14] in connection with spectral operators; [9] for a functional calculus of decomposable operators). In this case, one also has

$$
\boldsymbol{P}(\Gamma(\boldsymbol{T}))_{j, k}=\int_{\mathbb{R}}^{\oplus} P(\Gamma(T(t)))_{j, k} d t, \quad j, k \in\{1,2\} .
$$

We recall once more our convention throughout this manuscript that boldface symbols, such as $\boldsymbol{T}$, denote operators in the Hilbert space $L^{2}(\mathbb{R} ; \mathcal{H})$ associated with the direct integral over the family $\{T(t)\}_{t \in \mathbb{R}}$ as depicted in (3.14) (as opposed to our choice of notation $\mathcal{T}$ in the context of (3.10)).

If $T(t) \in \mathcal{B}(\mathcal{H}), t \in \mathbb{R}$, then

$$
\mathcal{T} \in \mathcal{B}\left(L^{2}(\mathbb{R} ; \mathcal{H})\right) \Longleftrightarrow \operatorname{esssup}_{t \in \mathbb{R}}\|T(t)\|_{\mathcal{B}(\mathcal{H})}<\infty,
$$

in particular, if $\mathcal{T} \in \mathcal{B}\left(L^{2}(\mathbb{R} ; \mathcal{H})\right)$, then

$$
\|\mathcal{T}\|_{\mathcal{B}\left(L^{2}(\mathbb{R} ; \mathcal{H})\right)}=\operatorname{esssup}_{t \in \mathbb{R}}\|T(t)\|_{\mathcal{B}(\mathcal{H})} .
$$

We recall the following results of Nussbaum [17] (in fact, he deals with the more general situation where the constant fiber space $\mathcal{H}$ is replaced by a measurable family of Hilbert spaces $\left.\{\mathcal{H}(t)\}_{t \in \mathbb{R}}\right)$ :

Lemma 3.4 (Nussbaum [17]). Assume Hypothesis 3.1 and suppose in addition that the family $\{T(t)\}_{t \in \mathbb{R}}$ is weakly measurable. Define $\mathcal{T}$ according to (3.10). Then $\mathcal{T}$ is a closed, decomposable operator in $L^{2}(\mathbb{R} ; \mathcal{H})=\int_{\mathbb{R}}^{\oplus} \mathcal{H} d t$. Thus, there exists an $N$-measurable family of closed operators $\{\widehat{T}(t)\}_{t \in \mathbb{R}}$ in $\mathcal{H}$ such that

$$
\mathcal{T}=\int_{\mathbb{R}}^{\oplus} \widehat{T}(t) d t
$$

and

$$
\widehat{T}(t) \subseteq T(t) \text { for a.e. } t \in \mathbb{R} .
$$

We note that it is not known if $\widehat{T}(t)$ in Lemma 3.4 are densely defined for a.e. $t \in \mathbb{R}$ in $\mathcal{H}$ (in which case also $\mathbf{T}$ would be densely defined in $L^{2}(\mathbb{R} ; \mathcal{H}$ ); see also Remark 4.7).

Theorem 3.5 (Nussbaum [17]). Assume Hypothesis 3.1 and suppose in addition that the family $\{T(t)\}_{t \in \mathbb{R}}$ is $N$-measurable. Then the following assertions hold:

(i) $\boldsymbol{T}=\int_{\mathbb{R}}^{\oplus} T(t) d t$ is densely defined and closed in $L^{2}(\mathbb{R} ; \mathcal{H})=\int_{\mathbb{R}}^{\oplus} \mathcal{H} d t$ and

$$
\boldsymbol{T}^{*}=\int_{\mathbb{R}}^{\oplus} T(t)^{*} d t, \quad|\boldsymbol{T}|=\int_{\mathbb{R}}^{\oplus}|T(t)| d t .
$$

(ii) $\boldsymbol{T}$ is symmetric (resp., self-adjoint, or normal) if and only if $T(t)$ is symmetric (resp., self-adjoint, or normal) for a.e. $t \in \mathbb{R}$.

(iii) $\operatorname{ker}(\boldsymbol{T})=\{0\}$ if and only if $\operatorname{ker}(T(t))=\{0\}$ for a.e. $t \in \mathbb{R}$. In addition, if $\operatorname{ker}(\boldsymbol{T})=\{0\}$, then $\left\{T(t)^{-1}\right\}_{t \in \mathbb{R}}$ is $N$-measurable and

$$
\boldsymbol{T}^{-1}=\int_{\mathbb{R}}^{\oplus} T(t)^{-1} d t .
$$

(iv) If $\boldsymbol{T}$ is self-adjoint in $L^{2}(\mathbb{R} ; \mathcal{H})$, then $\boldsymbol{T} \geqslant 0$ if and only if $T(t) \geqslant 0$ for a.e. $t \in \mathbb{R}$. 
(v) If $\boldsymbol{T}$ is normal in $L^{2}(\mathbb{R} ; \mathcal{H})$, then

$$
p(\boldsymbol{T})=\int_{\mathbb{R}}^{\oplus} p(T(t)) d t
$$

for any polynomial $p$.

(vi) Let $S(t), t \in \mathbb{R}$, be densely defined, closed operators in $\mathcal{H}$ and assume that the family $\{S(t)\}_{t \in \mathbb{R}}$ is $N$-measurable and $\boldsymbol{S}=\int_{\mathbb{R}}^{\oplus} S(t) d t$. Then $\boldsymbol{T} \subseteq \boldsymbol{S}$ if and only if $T(t) \subseteq S(t)$ for a.e. $t \in \mathbb{R}$.

Since $N$-measurability is a crucial hypothesis in Theorem 3.5, we emphasize Remark 3.3 (iii) which represents necessary and sufficient conditions which seem verifiable in practical situations. In addition, we note the following result:

Lemma 3.6. Assume Hypothesis 3.1 and suppose that

$$
\{T(t)\}_{t \in \mathbb{R}}, \quad\left\{\left(|T(t)|^{2}+I_{\mathcal{H}}\right)^{-1}\right\}_{t \in \mathbb{R}}, \quad \text { and }\left\{T(t)\left(|T(t)|^{2}+I_{\mathcal{H}}\right)^{-1}\right\}_{t \in \mathbb{R}}
$$

are weakly measurable. Then $\{T(t)\}_{t \in \mathbb{R}}$ is $N$-measurable.

Proof. Since $T(t)\left(|T(t)|^{2}+I_{\mathcal{H}}\right)^{-1} \in \mathcal{B}(\mathcal{H}), t \in \mathbb{R}$, and

$$
\left(T(t)\left(|T(t)|^{2}+I_{\mathcal{H}}\right)^{-1}\right)^{*}=T(t)^{*}\left(\left|T(t)^{*}\right|^{2}+I_{\mathcal{H}}\right)^{-1}, \quad t \in \mathbb{R},
$$

one concludes that $\left\{T(t)^{*}\left(\left|T(t)^{*}\right|^{2}+I_{\mathcal{H}}\right)^{-1}\right\}_{t \in \mathbb{R}}$ is weakly measurable too. Thus, for each $g \in \mathcal{H},\left\{T(t)^{*}\left(\left|T(t)^{*}\right|^{2}+I_{\mathcal{H}}\right)^{-1} g\right\}_{t \in \mathbb{R}}$ is weakly measurable in $\mathcal{H}$, in addition, $T(t)^{*}\left(\left|T(t)^{*}\right|^{2}+I_{\mathcal{H}}\right)^{-1} g \in \operatorname{dom}(T(t))$ for all $t \in \mathbb{R}$. Since $\{T(t)\}_{t \in \mathbb{R}}$ is weakly measurable, one thus concludes that

$$
\left\{T(t) T(t)^{*}\left(\left|T(t)^{*}\right|^{2}+I_{\mathcal{H}}\right)^{-1}\right\}_{t \in \mathbb{R}}=\left\{I_{\mathcal{H}}-\left(\left|T(t)^{*}\right|^{2}+I_{\mathcal{H}}\right)^{-1}\right\}_{t \in \mathbb{R}},
$$

and hence $\left\{\left(\left|T(t)^{*}\right|^{2}+I_{\mathcal{H}}\right)^{-1}\right\}_{t \in \mathbb{R}}$, is weakly measurable as well.

Next, we recall a result due to Lennon [13] on sums and products of decomposable operators (actually, Lennon considers a slightly more general situation). We use the usual conventions that if $A$ and $B$ are linear operators in $\mathcal{H}$ then

$$
\operatorname{dom}(A+B)=\operatorname{dom}(A) \cap \operatorname{dom}(B)
$$

and

$$
\operatorname{dom}(A B)=\{f \in \operatorname{dom}(B) \mid B f \in \operatorname{dom}(A)\} .
$$

Theorem 3.7 (Lennon [13]). Let $\boldsymbol{A}=\int_{\mathbb{R}}^{\oplus} A(t) d t$ and $\boldsymbol{B}=\int_{\mathbb{R}}^{\oplus} B(t) d t$ be closed decomposable operators in $L^{2}(\mathbb{R} ; \mathcal{H})=\int_{\mathbb{R}}^{\oplus} \mathcal{H} d t$ with the $N$-measurable families $\{A(t)\}_{t \in \mathbb{R}}$ and $\{B(t)\}_{t \in \mathbb{R}}$ in $\mathcal{H}$ satisfying Hypothesis 3.1. Then the following holds: (i) $\operatorname{dom}(\boldsymbol{A}+\boldsymbol{B})$ is dense in $L^{2}(\mathbb{R} ; \mathcal{H})$ if and only if $\operatorname{dom}(A(t) \cap B(t))$ is dense in $\mathcal{H}$ for a.e. $t \in \mathbb{R}$. In addition, $\boldsymbol{A}+\boldsymbol{B}$ is closable in $L^{2}(\mathbb{R} ; \mathcal{H})$ if and only if $A(t)+B(t)$ is closable in $\mathcal{H}$ for a.e. $t \in \mathbb{R}$. In this case the family $\{\overline{[A(t)+B(t)]}\}_{t \in \mathbb{R}}$ is $N$ measurable and

$$
\overline{\boldsymbol{A}+\boldsymbol{B}}=\int_{\mathbb{R}}^{\oplus} \overline{[A(t)+B(t)]} d t .
$$

(ii) $\operatorname{dom}(\boldsymbol{A B})$ is dense in $L^{2}(\mathbb{R} ; \mathcal{H})$ if and only if $\operatorname{dom}(A(t) B(t))$ is dense in $\mathcal{H}$ for a.e. $t \in \mathbb{R}$. In addition, $\boldsymbol{A} \boldsymbol{B}$ is closable in $L^{2}(\mathbb{R} ; \mathcal{H})$ if and only if $A(t) B(t)$ is 
closable in $\mathcal{H}$ for a.e. $t \in \mathbb{R}$. In this case the family $\{\overline{[A(t) B(t)]}\}_{t \in \mathbb{R}}$ is $N$-measurable and

$$
\overline{\boldsymbol{A B}}=\int_{\mathbb{R}}^{\oplus} \overline{[A(t) B(t)]} d t .
$$

We continue this discussion by reconsidering the special self-adjoint case in more detail which has important applications to periodic Schrödinger (resp., Jacobi, Dirac, CMV, etc.) operators (cf. [12, Chs. 3, 4], ][21, Sect. XIII.16]) and to random Hamiltonians in condensed matter physics (cf. [3, Ch. V, VII-IX ], [20, Chs. I, II, $\mathrm{VI}])$.

In Theorem 3.8 below, $E_{T}(\lambda), \lambda \in \mathbb{R}$, denotes the family of strongly right continuos spectral projections associated with the self-adjoint operator $T$ in $\mathcal{H}$.

Theorem 3.8. Let $T(t), t \in \mathbb{R}$, be self-adjoint operators in $\mathcal{H}$. Then the following items $(i)-(v)$ are equivalent:

(i) The family $\{T(t)\}_{t \in \mathbb{R}}$ is $N$-measurable.

(ii) For some (and hence for all) $z_{0} \in \mathbb{C} \backslash \mathbb{R},\left\{\left(T(t)-z_{0} I_{\mathcal{H}}\right)^{-1}\right\}_{t \in \mathbb{R}}$ is weakly measurable.

(iii) For all $\lambda \in \mathbb{R},\left\{E_{T(t)}(\lambda)\right\}_{t \in \mathbb{R}}$ is weakly measurable.

(iv) For all $s \in \mathbb{R},\left\{e^{i s T(t)}\right\}_{t \in \mathbb{R}}$ is weakly measurable.

(v) For all $F \in L^{\infty}(\mathbb{R} ; d x),\{F(T(t))\}_{t \in \mathbb{R}}$ is weakly measurable.

Proof. Since by hypothesis, $T(t)=T(t)^{*}, t \in \mathbb{R}$, equation (3.4) yields that

$N$-measurability of $\{T(t)\}_{t \in \mathbb{R}}$ is equivalent to weak measurability of

$$
\left\{\left(T(t)^{2}+I_{\mathcal{H}}\right)^{-1}\right\}_{t \in \mathbb{R}} \text { and }\left\{T(t)\left(T(t)^{2}+I_{\mathcal{H}}\right)^{-1}\right\}_{t \in \mathbb{R}} .
$$

To show the equivalence of items $(i)$ and $(i i)$ we will employ the identities

$$
\begin{aligned}
\left(T(t)^{2}+I_{\mathcal{H}}\right)^{-1} & =\left(T(t)-i I_{\mathcal{H}}\right)^{-1}\left(T(t)+i I_{\mathcal{H}}\right)^{-1}, \\
T(t)\left(T(t)^{2}+I_{\mathcal{H}}\right)^{-1} & =\left(T(t)-i I_{\mathcal{H}}\right)^{-1}-i\left(T(t)^{2}+I_{\mathcal{H}}\right)^{-1} \\
& =\left(T(t)+i I_{\mathcal{H}}\right)^{-1}+i\left(T(t)^{2}+I_{\mathcal{H}}\right)^{-1} .
\end{aligned}
$$

Assuming that the family $\{T(t)\}_{t \in \mathbb{R}}$ is $N$-measurable, the identity

$$
\left(T(t) \pm i I_{\mathcal{H}}\right)^{-1}=T(t)\left(T(t)^{2}+I_{\mathcal{H}}\right)^{-1} \mp i\left(T(t)^{2}+I_{\mathcal{H}}\right)^{-1},
$$

then proves that the families $\left\{\left(T(t) \pm i I_{\mathcal{H}}\right)^{-1}\right\}_{t \in \mathbb{R}}$ are weakly measurable. Using the resolvent equation for $T(t)$ and the fact that by (3.6), products of families of weakly measurable bounded operators are weakly measurable, one obtains that for all $z \in \mathbb{C} \backslash \mathbb{R}$, the family $\left\{\left(T(t)-z I_{\mathcal{H}}\right)^{-1}\right\}_{t \in \mathbb{R}}$ is weakly measurable. In particular, this proves that $(i)$ implies $(i i)$. Using once more the fact that products of families of weakly measurable bounded operators are weakly measurable, combining (3.30) and (3.31) immediately yields that $(i i)$ implies $(i)$.

The equivalence of items $(i i i),(i v)$, and $(v)$ with item $(i i)$ is familiar from the theory of random Hamiltonians (cf., e.g., [3, Sect. 5.1] and [11]) and follows from the facts that for all $f, g \in \mathcal{H}, t \in \mathbb{R}$,

$$
\begin{aligned}
& \left(f, e^{i s T(t)} g\right)_{\mathcal{H}}=\int_{\mathbb{R}} e^{i s \lambda} d\left(f, E_{T(t)}(\lambda) g\right)_{\mathcal{H}}, \quad s \in \mathbb{R}, \\
& \left(f,\left(T(t)-z I_{\mathcal{H}}\right)^{-1} g\right)_{\mathcal{H}}= \pm i \int_{0}^{\infty} e^{ \pm i z s}\left(f, e^{\mp i s T(t)} g\right)_{\mathcal{H}} d s, \quad \pm \operatorname{Im}(z)>0,
\end{aligned}
$$




$$
\begin{aligned}
\left(f, E_{T(t)}(\lambda) g\right)_{\mathcal{H}}=\lim _{\delta \downarrow 0} \lim _{\varepsilon \downarrow 0} \int_{-\infty}^{\lambda+\delta}( & f,\left[\left(H(t)-\left(\lambda^{\prime}+i \varepsilon\right) I_{\mathcal{H}}\right)^{-1}\right. \\
- & \left.\left.\left(H(t)-\left(\lambda^{\prime}-i \varepsilon\right) I_{\mathcal{H}}\right)^{-1}\right] g\right)_{\mathcal{H}} d \lambda^{\prime}, \quad \lambda \in \mathbb{R},
\end{aligned}
$$

and the fact that $F_{ \pm}=(|F| \pm F) / 2$ (away from a set of Lebesgue measure zero) is the limit of appropriate step functions $F_{n, \pm}, n \in \mathbb{N}$, in the $\|\cdot\|_{\infty}$-norm, with $0 \leqslant F_{n, \pm} \leqslant F_{ \pm}$. Finally, one uses the fact that pointwise limits of measurable functions are measurable.

The equivalence of items $(i)$ and $(i i)$ in Theorem 3.8 is of course well-known and used, for instance, in [21, Sect. XIII.16] to define measurability of $\{T(t)\}_{t \in \mathbb{R}}$ in the self-adjoint case.

We conclude with the following elementary yet useful result (cf., e.g., [3, Sect. 5.1] and [11]):

Lemma 3.9. Let $T(t)$ and $T_{n}(t), n \in \mathbb{N}$, be self-adjoint operators in $\mathcal{H}$ for each $t \in \mathbb{R}$. In addition, suppose that $\left\{T_{n}(t)\right\}_{t \in \mathbb{R}}$ is $N$-measurable in $\mathcal{H}$ for each $n \in \mathbb{N}$, and that for a.e. $t \in \mathbb{R}, T_{n}(t)$ converge in the weak (and hence strong) resolvent sense to $T(t)$ as $n \rightarrow \infty$. Then the family $\{T(t)\}_{t \in \mathbb{R}}$ is $N$-measurable in $\mathcal{H}$.

Proof. This follows from Theorem 3.8 (i), (ii) and

$$
\left(f,\left(T(t)-z I_{\mathcal{H}}\right)^{-1} g\right)_{\mathcal{H}}=\lim _{n \rightarrow \infty}\left(f,\left(T_{n}(t)-z I_{\mathcal{H}}\right)^{-1} g\right)_{\mathcal{H}}
$$

for all $f, g \in \mathcal{H}$, and a.e. $t \in \mathbb{R}$, using the fact that the a.e. limit of measurable functions is measurable (using the completeness of the Lebesgue measure).

\section{The Negative Answer to Nusssbaum's Question}

On p. 36 in his paper [17], Nussbaum asks whether the converse implication holds in (3.2). Given the implication in (3.2), this amounts of course to asking whether there is actually equivalence in (3.2). Explicitly, this then reads as follows:

- Is $N$-measurability of $\{T(t)\}_{t \in \mathbb{R}}$ equivalent to weak measurability of $\{T(t)\}_{t \in \mathbb{R}}$ ?

As described in (3.7), Nussbaum [17] proved the equivalence in (4.1) in the case of bounded operators $T(t) \in \mathcal{B}(\mathcal{H}), t \in \mathbb{R}$, and, as mentioned in Remark 3.3 (ii), he also proved (3.2), and hence that $N$-measurability of $\{T(t)\}_{t \in \mathbb{R}}$ implies weak measurability of $\{T(t)\}_{t \in \mathbb{R}}$. However, in the case of unbounded operators $T(t)$, the converse of this fact, and hence the question (4.1), appears to have been open for about 45 years now.

The principal purpose of this section is to answer Nussbaum's question and consider closely related questions of this type. In fact, we will provide several counterexamples with slightly varying degree of sophistication. In addition, we also derive some concrete scenarios in which $N$-measurability can be verified.

The following simple (and most likely, simplest) counterexample answers Nussbaum's question in the negative and hence demonstrates an interesting distinction between operators in $L^{2}(\mathbb{R} ; \mathcal{H})$ defined as in (3.10), and direct integrals as in (3.14):

Example 4.1. Let $T_{0}$ and $T_{1}$ be densely defined, closed, unbounded, symmetric operators in $\mathcal{H}$ satisfying

$$
T_{0} \subsetneq T_{1}
$$


Let $\mathfrak{E} \subset \mathbb{R}$ be a nonmeasurable subset of $\mathbb{R}$ (in the sense of Lebesgue measure) and introduce the linear operators

$$
\widetilde{T}(t)= \begin{cases}T_{0}, & t \in \mathfrak{E}, \\ T_{1}, & t \in \mathbb{R} \backslash \mathfrak{E},\end{cases}
$$

in $\mathcal{H}$. Then the family $\{\widetilde{T}(t)\}_{t \in \mathbb{R}}$ is weakly measurable, but not $N$-measurable.

Proof. Let $\{f(t)\}_{t \in \mathbb{R}}$ be a (weakly) measurable family of elements in $\mathcal{H}$ such that $f(t) \in \operatorname{dom}(\widetilde{T}(t))$ for all $t \in \mathbb{R}$. Then, using the fact that

$$
T_{0} \subset T_{1} \subseteq T_{1}^{*} \subset T_{0}^{*},
$$

one concludes that

$$
(g, \widetilde{T}(t) f(t))_{\mathcal{H}}=\left(T_{0} g, f(t)\right)_{\mathcal{H}}, \quad t \in \mathbb{R}, g \in \operatorname{dom}\left(T_{0}\right),
$$

is measurable, and since $\operatorname{dom}\left(T_{0}\right)$ is dense in $\mathcal{H}$, the family $\{\widetilde{T}(t)\}_{t \in \mathbb{R}}$ is weakly measurable by Remark $3.3(i)$.

Since by hypothesis, $T_{0} \subsetneq T_{1}$, one concludes that

$$
T_{0}^{*} T_{0} \neq T_{1}^{*} T_{1} .
$$

Arguing by contradiction, the equality $T_{0}^{*} T_{0}=T_{1}^{*} T_{1}$ would imply $\operatorname{dom}\left(T_{0}^{*} T_{0}\right)=$ $\operatorname{dom}\left(T_{1}^{*} T_{1}\right)$ and hence

$$
\operatorname{dom}\left(T_{0}\right)=\operatorname{dom}\left(\left|T_{0}\right|\right)=\operatorname{dom}\left(\left|T_{1}\right|\right)=\operatorname{dom}\left(T_{1}\right),
$$

where we used $\operatorname{dom}(T)=\operatorname{dom}(|T|)$ for any densely defined, closed operator $T$ in $\mathcal{H}$ and [27, Theorem 9.4(b)] (a consequence of Heinz's inequality) to obtain $\operatorname{dom}\left(\left|T_{0}\right|\right)=\operatorname{dom}\left(\left|T_{1}\right|\right)$. However, (4.7) contradicts hypothesis (4.2).

In addition, there exists $0 \neq h \in \mathcal{H}$ such that

$$
\left(h,\left(T_{0}^{*} T_{0}+I_{\mathcal{H}}\right)^{-1} h\right)_{\mathcal{H}} \neq\left(h,\left(T_{1}^{*} T_{1}+I_{\mathcal{H}}\right)^{-1} h\right)_{\mathcal{H}} .
$$

Indeed, if no such $0 \neq h \in \mathcal{H}$ existed, that is, if

$$
\left(k,\left(T_{0}^{*} T_{0}+I_{\mathcal{H}}\right)^{-1} k\right)_{\mathcal{H}}=\left(k,\left(T_{1}^{*} T_{1}+I_{\mathcal{H}}\right)^{-1} k\right)_{\mathcal{H}} \text { for all } k \in \mathcal{H},
$$

one arrives at

$$
\left(k_{1},\left(T_{0}^{*} T_{0}+I_{\mathcal{H}}\right)^{-1} k_{2}\right)_{\mathcal{H}}=\left(k_{1},\left(T_{1}^{*} T_{1}+I_{\mathcal{H}}\right)^{-1} k_{2}\right)_{\mathcal{H}} \text { for all } k_{1}, k_{2} \in \mathcal{H}
$$

since for any densely defined, closed, linear operator $T$ in $\mathcal{H}$, the sequilinear form $\left(k_{1},\left(T^{*} T+I_{\mathcal{H}}\right)^{-1} k_{2}\right)_{\mathcal{H}}, k_{1}, k_{2} \in \mathcal{H}$, satisfies

$$
\begin{aligned}
&\left(k_{1},\left(T^{*} T+I_{\mathcal{H}}\right)^{-1} k_{2}\right)_{\mathcal{H}}= \frac{1}{4}\left[\left(\left(k_{1}+k_{2}\right),\left(T^{*} T+I_{\mathcal{H}}\right)^{-1}\left(k_{1}+k_{2}\right)\right)_{\mathcal{H}}\right. \\
&-\left(\left(k_{1}-k_{2}\right),\left(T^{*} T+I_{\mathcal{H}}\right)^{-1}\left(k_{1}-k_{2}\right)\right)_{\mathcal{H}} \\
&+i\left(\left(k_{1}-i k_{2}\right),\left(T^{*} T+I_{\mathcal{H}}\right)^{-1}\left(k_{1}-i k_{2}\right)\right)_{\mathcal{H}} \\
&\left.-i\left(\left(k_{1}+i k_{2}\right),\left(T^{*} T+I_{\mathcal{H}}\right)^{-1}\left(k_{1}+i k_{2}\right)\right)_{\mathcal{H}}\right], \\
& k_{1}, k_{2} \in \mathcal{H} .
\end{aligned}
$$

Equation (4.10) implies $T_{0}^{*} T_{0}=T_{1}^{*} T_{1}$, again contradicting hypothesis (4.2). Thus, one arrives at the validity of (4.8). 
Since nonmeasurability of $\mathfrak{E}$ is equivalent to nonmeasurability of its characteristic function $\chi_{\mathfrak{E}}$, one similarly infers that

$$
\left(h,\left((\widetilde{T}(t))^{*} \widetilde{T}(t)+I_{\mathcal{H}}\right)^{-1} h\right)_{\mathcal{H}}= \begin{cases}\left(h,\left(T_{0}^{*} T_{0}+I_{\mathcal{H}}\right)^{-1} h\right)_{\mathcal{H}}, & t \in \mathfrak{E}, \\ \left(h,\left(T_{1}^{*} T_{1}+I_{\mathcal{H}}\right)^{-1} h\right)_{\mathcal{H}}, & t \in \mathbb{R} \backslash \mathfrak{E},\end{cases}
$$

is nonmeasurable, implying that the family $\{\widetilde{T}(t)\}_{t \in \mathbb{R}}$ is not $N$-measurable by equation (3.4).

An elementary concrete situation, illustrating (4.2), (4.4), and (4.6), is obtained as follows:

Example 4.2. Given $\mathcal{H}=L^{2}((0,1) ; d x)$, one introduces

$$
\begin{array}{rr}
T_{0}=\frac{1}{i} \frac{d}{d x}, \quad \operatorname{dom}\left(T_{0}\right)=\left\{f \in L^{2}((0,1) ; d x) \mid f \in A C([0,1]) ; f(0)=f(1)=0 ;\right. \\
\left.f^{\prime} \in L^{2}((0,1) ; d x)\right\}, \\
T_{1}=\frac{1}{i} \frac{d}{d x}, \quad \operatorname{dom}\left(T_{1}\right)=\left\{f \in L^{2}((0,1) ; d x) \mid f \in A C([0,1]) ; f(0)=f(1) ;\right. \\
\left.f^{\prime} \in L^{2}((0,1) ; d x)\right\},
\end{array}
$$

where $A C([0,1])$ denotes the set of absolutely continuous functions on $[0,1]$. Then $T_{0}$ is densely defined, closed, symmetric, with deficiency indices $(1,1)$, and $T_{1}$ is self-adjoint,

$$
T_{0} \subsetneq T_{1}=T_{1}^{*} \subsetneq T_{0}^{*},
$$

where

$$
T_{0}^{*}=\frac{1}{i} \frac{d}{d x}, \quad \operatorname{dom}\left(T_{0}^{*}\right)=\left\{f \in L^{2}((0,1) ; d x) \mid f \in A C([0,1]) ; f^{\prime} \in L^{2}((0,1) ; d x)\right\} .
$$

(See, e.g., [19, p. 141-142] for a discussion of these facts.) Moreover, one verifies

$$
\begin{gathered}
T_{0}^{*} T_{0}=-\frac{d^{2}}{d x^{2}}, \quad \operatorname{dom}\left(T_{0}^{*} T_{0}\right)=\left\{f \in L^{2}((0,1) ; d x) \mid f, f^{\prime} \in A C([0,1]) ;\right. \\
\left.f(0)=f(1)=0 ; f^{\prime}, f^{\prime \prime} \in L^{2}((0,1) ; d x)\right\} \\
T_{1}^{*} T_{1}=T_{1}^{2}=-\frac{d^{2}}{d x^{2}}, \quad \operatorname{dom}\left(T_{1}^{*} T_{1}\right)=\left\{f \in L^{2}((0,1) ; d x) \mid f, f^{\prime} \in A C([0,1]) ;\right. \\
\left.f(0)=f(1), f^{\prime}(0)=f^{\prime}(1) ; f^{\prime}, f^{\prime \prime} \in L^{2}((0,1) ; d x)\right\},
\end{gathered}
$$

that is, $T_{0}^{*} T_{0} \geqslant \pi^{2} I_{L^{2}((0,1) ; d x)}$ represents the (self-adjoint) Dirichlet Laplacian on $[0,1]$, whereas $T_{1}^{*} T_{1} \geqslant 0$ represents the (self-adjoint) periodic Laplacian on $[0,1]$.

Next, recalling the fact that by Remark $3.3(i v), N$-measurability of $\{T(t)\}_{t \in \mathbb{R}}$ is equivalent to $N$-measurability of $\left\{T(t)^{*}\right\}_{t \in \mathbb{R}}$, we now address the following natural question:

- Is weak measurability of $\{T(t)\}_{t \in \mathbb{R}}$ equivalent to weak measurability of $\left\{T(t)^{*}\right\}_{t \in \mathbb{R}}$ ?

We will show that the answer to this question is also negative (and hence independently answering Nussbaum's question in the negative once again). 
Let $A_{0}$ be a densely defined, closed, symmetric operator in $\mathcal{H}$ with deficiency indices $(1,1)$. Let $A_{1}$ be a self-adjoint extension of $A_{0}$ in $\mathcal{H}$. Define the operators $T_{0}$ and $T_{1}$ by

$$
T_{0}=A_{0}+i I_{\mathcal{H}}, \quad T_{1}=A_{1}+i I_{\mathcal{H}}, \quad \operatorname{dom}\left(T_{j}\right)=\operatorname{dom}\left(A_{j}\right), j=0,1 .
$$

We note that $\operatorname{ker}\left(T_{1}^{*}\right)=\{0\}$. On the other hand, there exists a vector $e \in \operatorname{dom}\left(T_{0}^{*}\right)$, with $\|e\|_{\mathcal{H}}=1$, such that

$$
T_{0}^{*} e=0 .
$$

Since $T_{0} \subsetneq T_{1}$ one obtains $T_{1}^{*} \subsetneq T_{0}^{*}$ and hence

$$
e \notin \operatorname{dom}\left(T_{1}^{*}\right) \text {. }
$$

(Otherwise, $T_{1}^{*} e=T_{0}^{*} e=0$ would yield a contradiction.) Define the operator $T_{2}$ by

$$
T_{2} f=T_{1} f+\left(e, T_{1} f\right)_{\mathcal{H}} e, \quad f \in \operatorname{dom}\left(T_{2}\right)=\operatorname{dom}\left(T_{1}\right) .
$$

Since

$$
T_{2}=K T_{1} \text {, where } K=I_{\mathcal{H}}+(e, \cdot)_{\mathcal{H}} e=K^{*} \text {, }
$$

and $K, K^{-1} \in \mathcal{B}(\mathcal{H}), T_{2}$ is closed in $\mathcal{H}$ and

$$
T_{2}^{*}=T_{1}^{*} K
$$

Example 4.3. Let $\mathfrak{E} \subset \mathbb{R}$ be a nonmeasurable subset of $\mathbb{R}$ (in the sense of Lebesgue measure). Given the facts (4.20)-(4.24), let the family $\{\widetilde{T}(t)\}_{t \in \mathbb{R}}$ of closed, densely defined operators in $\mathcal{H}$ be given by

$$
\widetilde{T}(t)= \begin{cases}T_{1}^{*}, & t \in \mathfrak{E}, \\ T_{2}^{*}, & t \in \mathbb{R} \backslash \mathfrak{E} .\end{cases}
$$

Then the family $\{\widetilde{T}(t)\}_{t \in \mathbb{R}}$ is weakly measurable, but the family of adjoint operators $\left\{(\widetilde{T}(t))^{*}\right\}_{t \in \mathbb{R}}$ is not weakly measurable.

Proof. First one notes that

$$
(\widetilde{T}(t))^{*}= \begin{cases}T_{1}, & t \in \mathfrak{E}, \\ T_{2}, & t \in \mathbb{R} \backslash \mathfrak{E} .\end{cases}
$$

For any $g \in \operatorname{dom}\left(T_{0}\right)$ one has

$$
\left(e, T_{1} g\right)_{\mathcal{H}}=\left(e, T_{0} g\right)_{\mathcal{H}}=\left(T_{0}^{*} e, g\right)_{\mathcal{H}}=0,
$$

and

$$
T_{2} g=T_{1} g=T_{0} g .
$$

Next, let $\{f(t)\}_{t \in \mathbb{R}}$ be a (weakly) measurable family of elements in $\mathcal{H}$ such that $f(t) \in \operatorname{dom}(\widetilde{T}(t))$ for all $t \in \mathbb{R}$. Then for any $g \in \operatorname{dom}\left(T_{0}\right)$ one obtains by (4.27) and (4.29), that

$$
(g, \widetilde{T}(t) f(t))_{\mathcal{H}}=\left(T_{0} g, f(t)\right)_{\mathcal{H}}, \quad t \in \mathbb{R}
$$

so that $(g, \widetilde{T}(t) f(t))_{\mathcal{H}}, g \in \operatorname{dom}\left(T_{0}\right)$, is measurable. Hence, using $\overline{\operatorname{dom}\left(T_{0}\right)}=\mathcal{H}$ and Remark $3.3(i)$, one concludes the weak measurability of $\{\widetilde{T}(t)\}_{t \in \mathbb{R}}$.

Noting that $\operatorname{dom}\left((\widetilde{T}(t))^{*}\right)=\operatorname{dom}\left(T_{1}\right), t \in \mathbb{R}$, and since $\operatorname{ker}\left(T_{1}^{*}\right)=\{0\}$, there exists a vector $h_{0} \in \operatorname{dom}\left(T_{1}\right)$ such that $\left(e, T_{1} h_{0}\right)_{\mathcal{H}} \neq 0$. Then

$$
\left(e,(\widetilde{T}(t))^{*} h_{0}\right)_{\mathcal{H}}= \begin{cases}\left(e, T_{1} h_{0}\right)_{\mathcal{H}}, & t \in \mathfrak{E}, \\ 2\left(e, T_{1} h_{0}\right)_{\mathcal{H}}, & t \in \mathbb{R} \backslash \mathfrak{E},\end{cases}
$$


so that $\mathbb{R} \ni t \mapsto\left(e,(\widetilde{T}(t))^{*} h_{0}\right)_{\mathcal{H}}$ is not measurable. Thus, $\left\{(\widetilde{T}(t))^{*}\right\}_{t \in \mathbb{R}}$ is not weakly measurable.

Remark 4.4. In Example 4.3 one has

$$
\bigcap_{t \in \mathbb{R}} \operatorname{dom}(\widetilde{T}(t))=\operatorname{dom}\left(T_{1}^{*}\right) \cap \operatorname{dom}\left(T_{2}^{*}\right)
$$

and

$$
\begin{aligned}
\operatorname{dom}\left(T_{1}^{*}\right) & =\operatorname{dom}\left(T_{1}\right)=\operatorname{dom}\left(A_{1}\right) \\
\operatorname{dom}\left(T_{2}^{*}\right) & =\left\{g \in \mathcal{H} \mid\left[g+(e, g)_{\mathcal{H}} e\right] \in \operatorname{dom}\left(T_{1}^{*}\right)\right\}
\end{aligned}
$$

Thus,

$$
\begin{aligned}
\bigcap_{t \in \mathbb{R}} \operatorname{dom}(\widetilde{T}(t)) & =\operatorname{dom}\left(T_{1}^{*}\right) \cap \operatorname{dom}\left(T_{2}^{*}\right) \\
& =\left\{g \in \operatorname{dom}\left(T_{1}^{*}\right) \mid\left[g+(e, g)_{\mathcal{H}} e\right] \in \operatorname{dom}\left(T_{1}^{*}\right)\right\} \\
& =\left\{g \in \operatorname{dom}\left(T_{1}^{*}\right) \mid(e, g)_{\mathcal{H}} e \in \operatorname{dom}\left(T_{1}^{*}\right)\right\} \\
& =\left\{g \in \operatorname{dom}\left(T_{1}^{*}\right) \mid(e, g)_{\mathcal{H}}=0\right\},
\end{aligned}
$$

since $e \notin \operatorname{dom}\left(T_{1}^{*}\right)$ by (4.22). Hence,

$$
\operatorname{codim}\left(\overline{\bigcap_{t \in \mathbb{R}} \operatorname{dom}(\widetilde{T}(t))}\right)=1
$$

and so $\bigcap_{t \in \mathbb{R}} \operatorname{dom}(\widetilde{T}(t))$ is "almost dense". It cannot be dense, however, if one is interested in a family $\left\{(\widetilde{T}(t))^{*}\right\}_{t \in \mathbb{R}}$ that is not weakly measurable as the next statement shows:

Lemma 4.5. Suppose that the family $\{T(t)\}_{t \in \mathbb{R}}$ of closed, densely defined operators in $\mathcal{H}$ is weakly measurable. Assume in addition, that

$$
\overline{\bigcap_{t \in \mathbb{R}} \operatorname{dom}(T(t))}=\mathcal{H} .
$$

Then the family $\left\{T(t)^{*}\right\}_{t \in \mathbb{R}}$ is weakly measurable.

Proof. Let $\{f(t)\}_{t \in \mathbb{R}}$ be a (weakly) measurable family of elements in $\mathcal{H}$ such that $f(t) \in \operatorname{dom}\left(T(t)^{*}\right)$ for all $t \in \mathbb{R}$. Then for any $g \in \bigcap_{t \in \mathbb{R}} \operatorname{dom}(T(t))$ one has

$$
\left(g, T(t)^{*} f(t)\right)_{\mathcal{H}}=(T(t) g, f(t))_{\mathcal{H}}, \quad t \in \mathbb{R},
$$

so that $\mathbb{R} \ni t \mapsto\left(g, T(t)^{*} f(t)\right)_{\mathcal{H}}$ is measurable by (3.1). In view of (4.37) and Remark 3.3 $(i)$, weak measurability of $\left\{T(t)^{*}\right\}_{t \in \mathbb{R}}$ follows.

Next, we show that condition (4.37) in Lemma 4.5 can be replaced by the condition that $\mathcal{T}$ is densely defined in $L^{2}(\mathbb{R} ; \mathcal{H})$ :

Lemma 4.6. Suppose that the family $\{T(t)\}_{t \in \mathbb{R}}$ of closed, densely defined operators in $\mathcal{H}$ is weakly measurable. Assume in addition, that $\mathcal{T}$, given by (3.10), is densely defined in $L^{2}(\mathbb{R} ; \mathcal{H})$. Then the family $\left\{T(t)^{*}\right\}_{t \in \mathbb{R}}$ is weakly measurable.

Proof. Let $\{f(t)\}_{t \in \mathbb{R}}$ be a (weakly) measurable family of elements in $\mathcal{H}$ such that $f(t) \in \operatorname{dom}\left(T(t)^{*}\right)$ for all $t \in \mathbb{R}$. Consider $g \in \mathcal{H}, n \in \mathbb{N}$, and

$$
\widetilde{g}_{n} \in L^{2}(\mathbb{R} ; \mathcal{H}) \text { with } \widetilde{g}_{n}(t)=\chi_{[-n, n]}(t) g, t \in \mathbb{R},
$$


(we recall that $\chi_{\mathcal{M}}$ denotes the characteristic function of a subset $\mathcal{M} \subset \mathbb{R}$ ). Since by hypothesis, $\overline{\operatorname{dom}(\mathcal{T})}=L^{2}(\mathbb{R} ; \mathcal{H})$, there exists a sequence $\left\{\widetilde{h}_{j}\right\}_{j \in \mathbb{N}} \subset \operatorname{dom}(\mathcal{T})$ such that

$$
\left\|\widetilde{g}_{n}-\widetilde{h}_{j}\right\|_{L^{2}(\mathbb{R} ; \mathcal{H})}^{2}=\int_{\mathbb{R}} d t\left\|\widetilde{g}_{n}(t)-\widetilde{h}_{j}(t)\right\|_{\mathcal{H}}^{2} \underset{j \rightarrow \infty}{\longrightarrow} 0 .
$$

Consequently, there exists a subsequence $\left\{\widetilde{h}_{j_{k}}\right\}_{k \in \mathbb{N}}$ of $\left\{\widetilde{h}_{j}\right\}_{j \in \mathbb{N}}$ such that for a.e. $t \in \mathbb{R}$

$$
\left\|\widetilde{g}_{n}(t)-\widetilde{h}_{j_{k}}(t)\right\|_{\mathcal{H}} \underset{k \rightarrow \infty}{\longrightarrow} 0
$$

and hence for a.e. $t \in[-n, n]$,

$$
\left\|g-\widetilde{h}_{j_{k}}(t)\right\|_{\mathcal{H}} \underset{k \rightarrow \infty}{\longrightarrow} 0
$$

As a result, for a.e. $t \in \mathbb{R}$,

$$
\begin{aligned}
\chi_{[-n, n]}(t)\left(T(t) \widetilde{h}_{j_{k}}(t), f(t)\right)_{\mathcal{H}}=\chi_{[-n, n]}(t)\left(\widetilde{h}_{j_{k}}(t), T(t)^{*} f(t)\right)_{\mathcal{H}} \\
\underset{k \rightarrow \infty}{\longrightarrow} \chi_{[-n, n]}(t)\left(h, T(t)^{*} f(t)\right)_{\mathcal{H}} .
\end{aligned}
$$

Since $h \in \mathcal{H}$ was arbitrary, this yields that $\left\{\chi_{[-n, n]}(t) T(t)^{*} f(t)\right\}_{t \in \mathbb{R}}$ is weakly measurable in $\mathcal{H}$. Since also $n \in \mathbb{N}$ was arbitrary, one concludes that $\left\{T(t)^{*} f(t)\right\}_{t \in \mathbb{R}}$ is weakly measurable in $\mathcal{H}$, which yields the weak measurability of $\left\{T(t)^{*}\right\}_{t \in \mathbb{R}}$.

Remark 4.7. Lemma 4.6 shows that if $\left\{T(t)^{*}\right\}_{t \in \mathbb{R}}$ is not weakly measurable, then $\mathcal{T}$ is not densely defined. In other words, Example 4.3 also generates an example of a non-densely defined operator $\mathcal{T}$ in $L^{2}(\mathbb{R} ; \mathcal{H})$.

The following result further clarifies the issue of density of the domain of $\mathcal{T}$.

Lemma 4.8. Let $\{T(t)\}_{t \in \mathbb{R}}$ be a weakly measurable family of densely defined, closed operators in $\mathcal{H}$ and assume that the linear operator $\mathcal{T}$ on $L^{2}(\mathbb{R} ; \mathcal{H})$ is defined as in (3.10). If

$$
\mathcal{D}=\left\{f \in L^{2}(\mathbb{R} ; \mathcal{H}) \mid f(t) \in \operatorname{dom}(T(t)) \text { for a.e. } t \in \mathbb{R}\right\},
$$

then

$$
\overline{\mathcal{D}}=\overline{\operatorname{dom}(\mathcal{T})} .
$$

Proof. Clearly $\operatorname{dom}(\mathcal{T}) \subseteq \mathcal{D}$, hence it suffices to prove that

$$
\mathcal{D} \subseteq \overline{\operatorname{dom}(\mathcal{T})} .
$$

If $f \in \mathcal{D}$, then the family $\{T(t) f(t)\}_{t \in \mathbb{R}}$ is measurable, hence the function $\mathbb{R} \ni$ $t \mapsto\|T(t) f(t)\|_{\mathcal{H}}$ is measurable too. The latter fact implies that the sets

$$
E_{n}=\left\{t \in \mathbb{R} \mid\|T(t) f(t)\|_{\mathcal{H}} \leqslant n\right\} \cap[-n, n], \quad n \in \mathbb{N},
$$

are measurable. In addition, one notes that $E_{n} \subseteq E_{n+1}, n \in \mathbb{N}$, and $\mathbb{R}=\bigcup_{n \in \mathbb{N}} E_{n}$. Define

$$
f_{n}(t)= \begin{cases}f(t), & t \in E_{n}, \\ 0, & t \in \mathbb{R} \backslash E_{n},\end{cases}
$$

then $f_{n} \in \mathcal{D}, n \in \mathbb{N}$, and

$$
\left\|f-f_{n}\right\|_{L^{2}(\mathbb{R} ; \mathcal{H})}^{2}=\int_{\mathbb{R} \backslash E_{n}}\|f(t)\|_{\mathcal{H}}^{2} d t \underset{n \rightarrow \infty}{\longrightarrow} 0 .
$$


Moreover, $f_{n} \in \operatorname{dom}(\boldsymbol{T})$ since

$$
\int_{\mathbb{R}}\left\|T(t) f_{n}(t)\right\|_{\mathcal{H}}^{2} d t=\int_{E_{n}}\|T(t) f(t)\|_{\mathcal{H}}^{2} d t \leqslant 2 n^{3} .
$$

Thus $\mathcal{D} \subseteq \overline{\operatorname{dom}(\mathcal{T})}$.

Corollary 4.9. Let $\{T(t)\}_{t \in \mathbb{R}}$ be a weakly measurable family of densely defined, closed operators in $\mathcal{H}$ and assume that the linear operator $\mathcal{T}$ on $L^{2}(\mathbb{R} ; \mathcal{H})$ is defined as in (3.10). Then $\mathcal{T}$ is densely defined if the linear subspace $\mathcal{D}$ of $L^{2}(\mathbb{R} ; \mathcal{H})$ given by $(4.44)$ is dense in $L^{2}(\mathbb{R} ; \mathcal{H})$.

Strengthening Example 4.1, we now provide an example of operators $\{T(t)\}_{t \in \mathbb{R}}$ such that (4.1) is violated and, in adddition, $T(t)$ are self-adjoint in $\mathcal{H}$ for all $t \in \mathbb{R}$ :

Example 4.10. Assume that $T_{0}$ is a densely defined, closed, symmetric operator with deficiency indices $(1,1)$ in $\mathcal{H}$, and let $T_{1}, T_{2}, T_{1} \neq T_{2}$, be two different selfadjoint extensions of $T_{0}$ in $\mathcal{H}$. Suppose that $\mathfrak{E} \subset \mathbb{R}$ is a nonmeasurable subset of $\mathbb{R}$ (in the sense of Lebesgue measure). Let $\{\widetilde{T}(t)\}_{t \in \mathbb{R}}$ be the family of closed, densely defined operators in $\mathcal{H}$ given by

$$
\widetilde{T}(t)= \begin{cases}T_{1}, & t \in \mathfrak{E} \\ T_{2}, & t \in \mathbb{R} \backslash \mathfrak{E} .\end{cases}
$$

Then the family $\{\widetilde{T}(t)\}_{t \in \in \mathbb{R}}$ of self-adjoint operators is weakly measurable, but not $N$-measurable.

Proof. We argue as in Example 4.1. Consider a (weakly) measurable family of elements $\{f(t)\}_{t \in \mathbb{R}}$ in $\mathcal{H}$ such that $f(t) \in \operatorname{dom}(\widetilde{T}(t))$ for all $t \in \mathbb{R}$.

Then for any $g \in \operatorname{dom}\left(T_{0}\right)$ one has

$$
(g, \widetilde{T}(t) f(t))_{\mathcal{H}}=\left(T_{0} g, f(t)\right)_{\mathcal{H}}, \quad t \in \mathbb{R}
$$

and hence $\mathbb{R} \ni t \mapsto(g, \widetilde{T}(t) f(t))_{\mathcal{H}}$ is measurable. Since $\operatorname{dom}\left(T_{0}\right)$ is dense in $\mathcal{H}$, Remark 3.3 (i) implies weak measurability of $\{\widetilde{T}(t)\}_{t \in \mathbb{R}}$.

On the other hand, as in Example 4.1, there exist elements $h, k \in \mathcal{H}$ such that

$$
\alpha_{1}=\left(h,\left(T_{1}^{2}+I\right)^{-1} k\right)_{\mathcal{H}} \neq\left(h,\left(T_{2}^{2}+I\right)^{-1} k\right)_{\mathcal{H}}=\alpha_{2} .
$$

Then the function

$$
\left(h,\left((\widetilde{T}(t))^{*} \widetilde{T}(t)+I_{\mathcal{H}}\right)^{-1} k\right)_{\mathcal{H}}= \begin{cases}\alpha_{1}, & t \in \mathfrak{E}, \\ \alpha_{2}, & t \in \mathbb{R} \backslash \mathfrak{E},\end{cases}
$$

is nonmeasurable. Thus, the family $\{\widetilde{T}(t)\}_{t \in \mathbb{R}}$ is not $N$-measurable.

We conclude with a positive result characterizing $N$-measurability in terms of resolvents (cf. also Theorem $3.8(i)$ and $(i i)$ ):

Theorem 4.11. Let $\{T(t)\}_{t \in \mathbb{R}}$ be a family of densely defined, closed operators in $\mathcal{H}$. Suppose that there exists a measurable function $\mathbb{R} \ni t \mapsto \alpha(t) \in \mathbb{C}$ such that

$$
\alpha(t) \in \rho(T(t)) \text { for a.e. } t \in \mathbb{R},
$$

and, in addition, the family of bounded operators $\left\{\left(T(t)-\alpha(t) I_{\mathcal{H}}\right)^{-1}\right\}_{t \in \mathbb{R}}$ is weakly measurable. Then the family $\{T(t)\}_{t \in \mathbb{R}}$ is $N$-measurable. 
Proof. Since $\left(T(t)-\alpha(t) I_{\mathcal{H}}\right)^{-1} \in \mathcal{B}(\mathcal{H}), t \in \mathbb{R}$, weak measurability of the family $\left\{\left(T(t)-\alpha(t) I_{\mathcal{H}}\right)^{-1}\right\}_{t \in \mathbb{R}}$ is equivalent to its $N$-measurability by (3.7). By Theorem $3.5($ iii $)$, the family $\left\{T(t)-\alpha(t) I_{\mathcal{H}}\right\}_{t \in \mathbb{R}}$ is $N$-measurable. Since $\alpha(t) I_{\mathcal{H}} \in \mathcal{B}(\mathcal{H})$ and $T(t)$ is closed for all $t \in \mathbb{R}$, Theorem $3.7(i)$ implies that $\left[T(t)-\alpha(t) I_{\mathcal{H}}\right]+\alpha(t) I_{\mathcal{H}}$ is densely defined and closable for a.e. $t \in \mathbb{R}$, and additionally,

$$
\overline{\left[T(t)-\alpha(t) I_{\mathcal{H}}\right]+\alpha(t) I_{\mathcal{H}}}=\overline{T(t)}=T(t) \text { is } N \text {-measurable. }
$$

Acknowledgments. We are indebted to Vladimir Chilin, Ben de Pagter, Nigel Kalton, and Konstantin Makarov for helpful discussions. In particular, Nigel Kalton suggested looking for examples of the type Example 4.10 with self-adjoint fiber operators $\widetilde{T}(t), t \in \mathbb{R}$.

\section{REFERENCES}

[1] W. Arendt, C. K. Batty, M. Hieber, F. Neubrander, Vector-Valued Laplace Transforms and Cauchy Transforms, Monographs in Mathematics, Vol. 96, Birkhäuser, Basel, 2001.

[2] T. Ya. Azizov, J. Behrndt, P. Jonas, and C. Trunk, Compact and finite rank perturbations of closed linear operators and relations in Hilbert space, Integral Eq. Operator Th. 63, 151-163 (2009).

[3] R. Carmona and J. Lacroix, Spectral Theory of Random Schrödinger Operators, Birkhäuser, Basel, 1990.

[4] T. R. Chow, A spectral theory for direct integrals of operators, Math. Ann. 188, 285-303 (1970).

[5] P. A. Deift, Applications of a commutation formula, Duke Math. J. 45, 267-310 (1978).

[6] J. Diestel and J. J. Uhl, Vector Measures, Mathematical Surveys, Vol. 15, Amer. Math. Soc., Providence, RI, 1977.

[7] J. Dixmier, Les Algèbres d'Opérateurs dans l'Espace Hilbertien (Algebrès de von Neumann), 2nd extended ed., Gauthier-Villars, Paris, 1969, Éditions Jaques Gabai, 1996.

[8] P. G. Dixon, Unbounded operator algebras, Proc. London Math. Soc. (3) 23, 53-69 (1971).

[9] F. Gilfeather, On a functional calculus for decomposable operators and applications to normal, operator-valued functions, Trans. Amer. Math. Soc. 176, 369-383 (1973).

[10] S. Hassi, Z. Sebestyén, H. S. V. De Snoo, and F. H. Szafraniec, A canonical decomposition for linear operators and linear relations, Acta Math. Hungar. 115, 281-307 (2007).

[11] W. Kirsch and F. Martinelli, On the ergodic properties of the spectrum of general random operators, J. reine angew. Math. 334, 141-156 (1982).

[12] P. Kuchment, Floquet Theory for Partial Differential Operators, Operator Theory: Advances and Applications, Vol. 60, Birkhäuser, Basel, 1993.

[13] M. J. J. Lennon, On Sums and Products of Unbounded Operators in Hilbert Space, Trans. Amer. Math. Soc. 198, 273-285 (1974).

[14] M. J. J. Lennon, Direct integral decomposition of spectral operators, Math. Ann. 207, 257-268 (1974).

[15] F. I. Mautner, Unitary representations of locally compact groups I, Ann. Math. (2) 51, 1-25 (1950).

[16] Y. Mezroui, Projection orthogonale sur le graphe d'une relation linèaire fermé, Trans. Amer. Math. Soc. 352, 2789-2800 (2000).

[17] A. E. Nussbaum, Reduction theory for unbounded closed operators in Hilbert space, Duke Math. J. 31, 33-44 (1964).

[18] R. Pallu de la Barrière, Décomposition des opérateurs non bornés dans les sommes continues d'espaces de Hilbert, Comptes Rendus Acad. Sci. Paris 232, 2071-2073 (1951).

[19] M. Reed and B. Simon, Methods of Modern Mathematical Physics. II: Fourier Analysis, Self-Adjointness, Academic Press, New York, 1975.

[20] L. Pastur and A. Figotin, Spectra of Random and Almost-Periodic Operators, Springer, Berlin, 1992. 
[21] M. Reed and B. Simon, Methods of Modern Mathematical Physics. IV: Analysis of Operators, Academic Press, New York, 1978.

[22] J. T. Schwartz, $W^{*}$-Algebras, Gordon and Breach, New York, 1967.

[23] M. H. Stone, On unbounded operators in Hilbert space, J. Indian Math. Soc. 15, 155-192 (1951).

[24] H. Takemoto, Decomposable operators in continuous fields of Hilbert spaces, Tôhoku Math. J. 27, 413-435 (1975).

[25] J. von Neumann, On rings of operators. Reduction theory, Ann. Math. (2) 50, 401-485 (1949).

[26] J. von Neumann, Functional Operators. Vol. II: The Geometry of Orthogonal Spaces, Ann. Math. Stud., Vol. 22, 2nd printing, Princeton University Press, Princeton, 1951.

[27] J. Weidmann, Linear Operators in Hilbert Spaces, Graduate Texts in Mathematics, Vol. 68, Springer, New York, 1980.

Department of Mathematics, University of Missouri, Columbia, MO 65211, USA

E-mail address: gesztesyf@missouri.edu

URL: http://www.math.missouri.edu/personnel/faculty/gesztesyf.html

Faculty of Mathematics and Computer Science, Nicholas Copernicus University, ul. Chopina 12/18, 87-100 Torun, Poland, and Institute of Mathematics, Polish Academy of Sciences. Śniadeckich Str. 8, 00-956 Warsaw, Poland

E-mail address: gomilko@mat.uni.torun.pl

School of Mathematics and Statistics, UnSW, Kensington, NSW 2052, Australia

E-mail address: f.sukochev@unsw.edu.au

Faculty of Mathematics and Computer Science, Nicholas Copernicus University, ul. Chopina 12/18, 87-100 Torun, Poland, and Institute of Mathematics, Polish Academy of Sciences. Śniadeckich Str. 8, 00-956 Warsaw, Poland

E-mail address: tomilov@mat.uni.torun.pl 